\title{
Invasive fungal disease in humans: are we aware of the real impact?
}

\author{
Carolina Firacative ${ }^{1 /+}$ \\ ${ }^{1}$ Universidad del Rosario, School of Medicine and Health Sciences, Studies in Translational Microbiology and Emerging Diseases (MICROS) \\ Research Group, Bogota, Colombia
}

Despite the medical advances and interventions to improve the quality of life of those in intensive care, people with cancer or severely immunocompromised or other susceptible hosts, invasive fungal diseases (IFD) remain severe and underappreciated causes of illness and death worldwide. Therefore, IFD continue to be a public health threat and a major hindrance to the success of otherwise life-saving treatments and procedures. Globally, hundreds of thousands of people are affected every year with Candida albicans, Aspergillus fumigatus, Cryptococcus neoformans, Pneumocystis jirovecii, endemic dimorphic fungi and Mucormycetes, the most common fungal species causing invasive diseases in humans. These infections result in morbidity and mortality rates that are unacceptable and represent a considerable socioeconomic burden. Raising the general awareness of the significance and impact of IFD in human health, in both the hospital and the community, is hence critical to understand the scale of the problem and to raise interest to help fighting these devastating diseases.

Key words: fungi - immunocompromised host - invasive fungal disease - risk factors

It is estimated that the fungal kingdom has six million species widely distributed in the environment. ${ }^{(1)}$ Among these, several hundred species of both yeasts and molds can affect humans in several ways. The direct ingestion of poisonous mushrooms causes mycetismus, which can result in gastrointestinal tract disorders or even death. In cases of mycotoxicosis, humans can be also poisoned by the ingestion of food contaminated with fungal toxins (mycotoxins), which affect the liver, kidneys and other vital organs..$^{(2)}$ Lastly, causing infection, fungi can be either primary or opportunistic pathogens of humans and the disease is called mycosis. Ranging from superficial infections of the hair, skin, nails or mucosal surfaces, which are usually benign, and other ailments including allergies, fungi can also cause invasive infections of internal organs that are progressive and lethal if not diagnosed and specifically treated..$^{(3,4)}$ To cause invasive disease in humans, however, fungi must meet four criteria: (i) the ability to grow at or above mammalian body temperature; (ii) the ability to reach internal tissues by penetrating or evading host barriers; (iii) the ability to lyse tissues and absorb their components; and (iv) the ability to evade host immune defences. ${ }^{(5)}$ As warmblooded animals with a highly sophisticated immune system, humans are naturally resistant to most invasive fungal diseases (IFD). As such, most cases of invasive disease occur in patients with an underlying serious illness or condition. Moreover, advances in medical care and life-saving treatments, which may lead to an impaired immune function, have increased the number of susceptible patients or people at-risk for fungal infections. ${ }^{(5)}$ Lastly, socioeconomic and geoecological characteristics, including the exposure to a high inoculum

doi: 10.1590/0074-02760200430

+ Corresponding author: cfiracative@gmail.com

(D) https://orcid.org/0000-0001-7547-5172

Received 21 August 2020

Accepted 24 September 2020 of fungi in their natural habitat, are also important elements that influence on the incidence and prevalence of fungal disease across the world. ${ }^{(6)}$

In this perspective article, the main risk factors of people to develop IFD are summarised, to aid recognising the impact of fungi on human health, an issue often underestimated and underappreciated. Considering that IFD are often disregarded during routine hospital care, it is very important to emphasise to microbiologists, diagnostic laboratory staff and clinicians, that not only the number but the diversity of patients at-risk for IFD are continuously rising, with mortality rates than remain unacceptably high. ${ }^{(3)}$ Additionally, most fungal infections are not reportable diseases, thus reliable information on local and global prevalence is not available. This review recalls the updated definition of IFD, collates recent epidemiological data and describes, in order of frequency, the most susceptible groups of people to acquire an IFD, namely those who undergo medical interventions, people with immunosuppression induced either by the treatment of an associated illnesses or by an underlying disease, people with bacterial or viral co-infections and immunocompetent individuals who acquire an IFD by environmental exposure.

\section{Invasive fungal diseases}

According to standard criteria, an invasive or systemic fungal disease is proven when tissue damage due to fungal elements is observed by histopathologic examination and/or when the aetiologic agent is isolated by culture from clinical sterile samples such as blood, tissue or cerebrospinal fluid. ${ }^{(7)}$ Infection can be initiated by invasion of fungal microbiota into the mucosa, by the inhalation of fungal spores from the environment or by direct inoculation, which lead to colonisation and dissemination. As clinical manifestations of IFD are not specific and the severity of the disease depends on the host's defences and immune response, a high degree of suspicion is needed for the early diagnosis and optimal management of these infections. ${ }^{(8)}$ 
IFD are an emerging problem worldwide, are generally very difficult to cure and the associated mortality remains very high depending on the pathogen and patient population. Recent studies have estimated that globally, fungal infections kill more than 1.5 million people per year, which is similar to the mortality due to tuberculosis and about three-times more than malaria ${ }^{(6)}$ However, the true burden of IFD is probably miscalculated because of the absence or availability of reliable, sensitive and universal diagnostic methods and because fungal infections are usually hindered by other diseases. Furthermore, when cases of fungal diseases are identified by a health provider, hospital or laboratory, they are not mandatory to be reported to public health departments; hence, most countries lack surveillance systems for IFD.

Among at-risk and immunocompromised patients, Candida albicans, Aspergillus fumigatus, Cryptococcus neoformans, Pneumocystis jirovecii, endemic dimorphic fungi and Mucormycetes remain the main fungal pathogens responsible for most cases of serious fungal diseases in the world (Tables I, II, Figure). ${ }^{(5,6,9,10)}$ While the ability of these fungi to cause disease depends on their virulence factors and pathogenic capacity, as well as on the interactions with the host and adaptation to different environments, the outcome of the infections is influenced by various others factors. The patients' underlying medical condition, the time for diagnosis, the choice of therapy, especially when fighting strains or species that are resistant to antifungal drugs, the capacity to achieve prompt and effective source control, and the adverse effects of antifungal drugs alone or concomitantly administered with other drugs, are factors that significantly contribute to IFD prognosis. ${ }^{(5)}$ Thus, identifying susceptible patients or those who are clinically suspected of having a fungal infection, along with an early empiric therapy or prophylaxis, are of paramount importance to decrease mortality and morbidity associated to IFD.

\section{Medical interventions}

The placement of central venous catheters and intravascular or intracranial devices plus the use of broadspectrum antibiotics, which are associated with medical interventions, are responsible for the vast majority of IFD acquired in hospital settings. Candidaemia, the most frequent life-threatening mycosis in the world, appears among the four most common causes of health care-associated bloodstream infections (BSI), with about $80 \%$ of cases occurring in the absence of evident immunosuppression but rather among patients with common iatrogenic and/or nosocomial conditions. ${ }^{(11,12)}$ Medical devices disrupt the normal skin barriers and provide direct access for the commensal yeasts of the genus Candida to the body's interior. In addition, it has been long recognised that antibiotics suppress the growth of normal bacterial microbiota, which favours the proliferation of Candida species, thus leading to colonisation and an increased risk of disseminated candidiasis. ${ }^{(12,13)}$ C. albicans (A in Figure) and other Candida species also have the ability to form biofilms on indwelling venous and bladder catheters, heart valves, joint replacements and even on different tissues in the host. Biofilms are a physical barrier intrinsically resistant to the host immune system and other environmental perturbations. ${ }^{(14)}$ The formation of biofilms additionally leads to upregulation of antifungal-resistance mechanisms, as well as the development of complex regulatory processes that favour even higher levels of antifungal resistance, most notably to azoles and polyenes. ${ }^{(15)}$ Directly related with medical interventions, nosocomial candidaemia is associated with prolonged stays in hospital and intensive care units, parenteral nutrition, as well as with improved survival of premature infants. ${ }^{(1)}$ Despite the broad use of antifungal prophylaxis, mostly fluconazole, which has shown to reduce the incidence of invasive candidiasis in patients who are at highest risk, almost 750,000 cases of this mycosis are estimated to occur yearly in the world. ${ }^{(6,12)}$ Although C. albicans is the global leading agent of candidaemia and other forms of invasive candidiasis, accounting for about half the amount of cases, the frequency of non-albicans species, mainly C. glabrata, C. parapsilosis, C. tropicalis and C. krusei is increasing, which is of concern when selecting empiric antifungal therapy, as non-albicans species have reduced susceptibility or are intrinsically resistance to azoles. ${ }^{(11)}$

Much less frequently, in both immunocompetent and immunocompromised individuals, fungi, including Candida spp., can be directly inoculated through neurosurgical procedures and contaminated devices or drug preparations. An unprecedented outbreak of fungal meningitis, for instance, was associated with parenteral injections of corticosteroids contaminated with $A$. fumigatus and Exserohilum rostratum, both environmental molds. ${ }^{(16,17)}$ Another saprophyte mold, Sarocladium kiliense, was isolated from several fungaemia cases associated with the administration of contaminated antinausea medication among oncology patients. ${ }^{(18,19)}$

\section{Treatment-induced immunosuppression}

Haematological malignancies, mainly acute leukaemia, account for most cases of IFD among patients immunosuppressed in the classical sense. Prolonged severe neutropenia, resulting from intense chemotherapy regimens and defined as more than 10 days with a peripheral blood absolute neutrophil count less than $500 / \mu \mathrm{L}$, is still the single major risk factor for invasive infection caused by the environmental mold A. fumigatus (B in Figure) (> 80\%

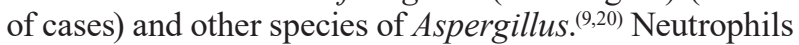
are known to be the primary host defence mechanism against Aspergillus infection, which commonly starts by the inhalation into and establishment of fungal spores in the lower respiratory tract. When mucosal barriers have been injured, neutrophils function in the innate immune response through the killing of conidia and hyphae by both oxidative and nonoxidative mechanisms. ${ }^{(21,22)}$ In neutropenic patients, therefore, neutrophil dysfunction represents an impairment to efficiently recruit and kill Aspergillus infectious elements. Worldwide, it is estimated that invasive aspergillosis affects annually more than 300,000 patients from almost 10 million at risk. ${ }^{(6)}$ Despite the availability of highly active antifungal treatment and the implementation of prevention strategies such as the introduction of mold-active prophylaxis and the reduction 
TABLE I

Major risk factors associated with the acquisition of an invasive fungal disease

\begin{tabular}{|c|c|c|}
\hline Risk factor & Specific conditions & Most common pathogens \\
\hline \multirow{3}{*}{ Medical intervention } & Catheters, intravascular or intracranial devices & Candida albicans, Candida spp. \\
\hline & Broad-spectrum antibiotics use & C. albicans, Candida spp. \\
\hline & $\begin{array}{l}\text { Neurosurgical procedures, } \\
\text { contaminated devices and drug preparations }\end{array}$ & Candida spp., saprophyte fungi \\
\hline \multirow{4}{*}{$\begin{array}{l}\text { Treatment-induced } \\
\text { immunosuppression }\end{array}$} & Severe prolonged neutropenia & Aspergillus fumigatus, Aspergillus spp. \\
\hline & Solid organ transplantation & $\begin{array}{l}\text { C. albicans, Candida spp., A. fumigatus, } \\
\text { Cryptococcus neoformans }\end{array}$ \\
\hline & Haematopoietic stem cell transplantation & $\begin{array}{l}\text { A. fumigatus, C. albicans, Candida spp., } \\
\text { Pneumocystis jirovecii, Mucormycetes }\end{array}$ \\
\hline & Biological agents & $\begin{array}{l}\text { Candida spp., Aspergillus spp., Cryptococcus spp., } \\
\text { P. jirovecii, dimorphic fungi, Mucormycetes }\end{array}$ \\
\hline \multirow{3}{*}{$\begin{array}{l}\text { Disease-induced } \\
\text { immunosuppression }\end{array}$} & HIV infection & $\begin{array}{c}\text { P. jirovecii, C. neoformans, } \\
\text { Histoplasma capsulatum, Talaromyces marneffei }\end{array}$ \\
\hline & Uncontrolled diabetes & Rhizopus oryzae, Mucormycetes \\
\hline & Chronic obstructive pulmonary disease & A. fumigatus \\
\hline \multirow{3}{*}{ Co-infection } & Tuberculosis & $\begin{array}{c}\text { A. fumigatus, Aspergillus niger, } H \text {. capsulatum, } \\
\text { C. neoformans, C. albicans }\end{array}$ \\
\hline & Cytomegalovirus & $\begin{array}{l}\text { P. jirovecii, Aspergillus spp., } \\
\text { non-Aspergillus molds }\end{array}$ \\
\hline & SARS-CoV-2 infection & A. fumigatus \\
\hline Environmental exposure & Trauma & R. oryzae, Mucormycetes \\
\hline
\end{tabular}

HIV: human immunodeficiency virus; SARS-CoV-2: severe acute respiratory syndrome coronavirus 2.

of exposure to Aspergillus spores, invasive aspergillosis in haemato-oncological patients is still associated with extremely high mortality rates $(60-70 \%)$. $^{(23,24)}$

Similarly, thousands of solid organ transplant (SOT) recipients in the world are at risk of IFD as a result of organ damage, neutropenia, administration of immunosuppressive drugs and surgical factors such as prolonged operation time, increased technical complexity and bleeding complications. In these patients, Candida spp., A. fumigatus and C. neoformans ( $\mathrm{C}$ in Figure) are reported as the most common fungal pathogens causing invasive disease, although the incidence of other molds, such as Fusarium spp. and dimorphic fungi, is increasing. ${ }^{(25,26)}$ In SOT recipients, however, the risk for and distribution of IFD depend on the organ transplanted. It has been reported that kidney transplant recipients had the lowest overall risk of IFD and lung transplant recipients are more commonly affected by Aspergillus spp. ${ }^{(26,27)}$ Survival of transplanted patients who developed an IFD also differs depending on the aetiological agent causing the infection, with candidaemia and other forms of invasive candidiasis generally accounting for higher number of deaths. ${ }^{(25,26)}$ While invasive candidiasis is generally an early complication of SOT, aspergillosis, cryptococcosis and other fungal infections tend to present later after transplantation. In addition, time to onset of IFD may also differ based on antifungal prophylaxis, site of infection or transplant type. ${ }^{(28)}$
Cytopaenia and administration of therapies to prevent and treat graft-versus-host disease (GvHD), which leads to an impaired cell immunity, are major risk factors of haematopoietic stem cell transplant (HSCT) recipients for acquiring IFD. ${ }^{(29,30)}$ Due to induced neutropenia and lack of adequate immune reconstitution, invasive aspergillosis has been noted to be the most commonly observed IFD in HSCT recipients, followed by invasive candidiasis, Pneumocystis pneumonia, mucormycosis (caused by Mucormycetes) and infections caused by other molds such as Fusarium and Scedosporium species. $(31,32)$ Gastrointestinal tract mucositis, defined as the disruption of the gastrointestinal tract mucosa, together with the presence of venous catheters in patients who require transfusion support, allow for gut and skin microbiota, including Candida spp., to invade and cause BSI. ${ }^{(30,32)}$ As in SOT recipients, IFD attributable mortality rates differ depending on the aetiological agent causing the infection, with non-Aspergillus mycosis and mucormycosis being responsible for most fatal cases in stem cell transplanted patients. $^{(31,33)}$ Donor type, GvHD grade, conditioning regimens, supportive care strategies and therapy timing are factors that also influence the risk for IFD in HSCT recipients. ${ }^{(30)}$ Clearly, invasive mycoses hamper the success of the transplantation and continue to be major threats to patients undergoing SOT and HSCT.

Biologic agents, such as antagonists of the tumour necrosis factor (TNF)- $\alpha$, which are used as therapeutic 
TABLE II

Estimated incidence and mortality rates of the most frequent invasive fungal diseases affecting humans

\begin{tabular}{lccc}
\hline Mycosis & Main aetiologic agent & Cases per year & Mortality rate (\%) \\
\hline Invasive candidiasis & Candida albicans & $\sim 750,000^{(6)}$ & $\sim 40^{(74)}$ \\
\hline Invasive aspergillosis & Aspergillus fumigatus & $>300,000^{(6)}$ & $30-70^{(23,24)}$ \\
\hline Pneumocystis pneumonia & Pneumocystis jirovecii & $>400,000^{(10)}$ & $10-60^{(38)}$ \\
\hline Cryptococcal meningitis & Cryptococcus neoformans & $\sim 225,000^{(44)}$ & $15-50^{(74)}$ \\
\hline Disseminated histoplasmosis & Histoplasma capsulatum & $\sim 100,000^{(6)}$ & $10-60^{(42)}$ \\
\hline Mucormycosis & Rhizopus oryzae & $>10,000^{(6)}$ & $35-100^{(49)}$ \\
\hline
\end{tabular}

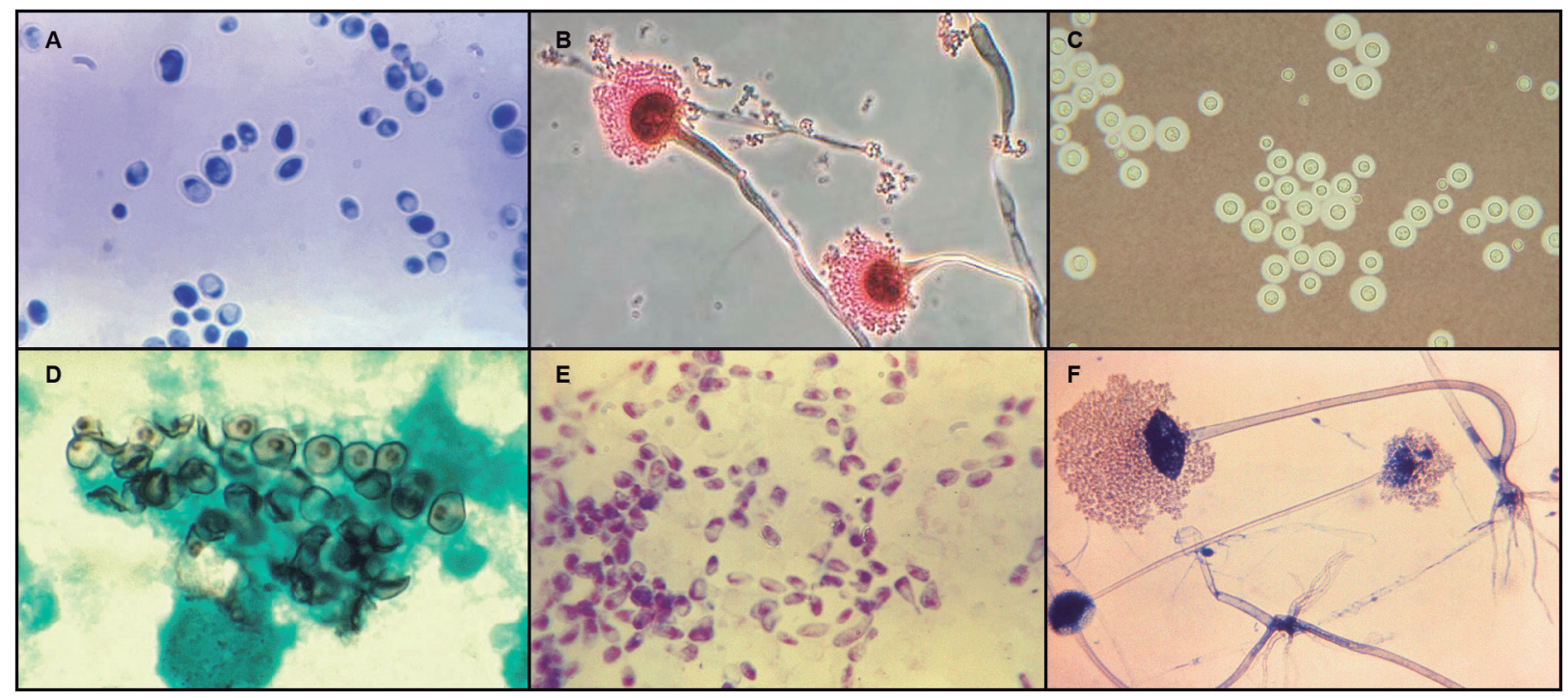

Photomicrographs of the most common fungal pathogens causing invasive diseases in humans. A: Candida albicans in its yeast-phase; B: filamentous conidiophores of Aspergillus fumigatus; C: encapsulated yeasts of Cryptococcus neoformans; D: Pneumocystis jirovecii cysts; E: Histoplasma capsulatum in its yeast phase; F: reproductive sporangia of Rhizopus oryzae. Images are not copyrighted and were obtained from the public domain of the Public Health Image Library (PHIL) from the Centers for Disease Control and Prevention (CDC) (phil.cdc.gov). ${ }^{(73)}$

approaches for autoimmune conditions including rheumatoid arthritis, juvenile idiopathic arthritis, psoriatic arthritis, ankylosing spondylitis, plaque psoriasis and inflammatory bowel diseases directly or indirectly impact the immune response against fungal pathogens, mainly by affecting inflammatory processes, thus increasing the risk of IFD. ${ }^{(34,35)}$ The immune response against fungal pathogens can be impaired by the blockade of TNF- $\alpha$, which leads to reduced cytokine production, impairment of monocyte recruitment, prevention of granuloma formation and apoptotic and nonapoptotic death of cells expressing TNF receptors. ${ }^{(34)}$ Although baseline predisposition of patients for fungal infections due to the autoimmune disorders, concomitant immunosuppressive therapies and geographical location need to be considered, Aspergillus, Candida and Cryptococcus infections are generally associated with the use TNF- $\alpha$ inhibitors. A study reported that among people receiving these biologic agents, candidiasis was the most frequent mycosis followed by histoplasmosis, aspergillosis, cryptococcosis and coccidioidomycosis, with incidences that ranged between 5.1 to 13.3 cases per 100,000 persons treated, over a 4.75-year period.( ${ }^{(36)}$ Other studies, however, reported $P$. jirovecii, a unculturable yeast-like fungus, as the most common nonviral opportunistic infection, with 16 cases among 33,324 users of TNF- $\alpha$ inhibitors. ${ }^{(37)}$ More rarely, agents of mucormycosis, other dimorphic fungi and non-Aspergillus molds have been reported in patients on active biologic therapy. ${ }^{(34)}$

\section{Disease-induced immunosuppression}

Defects in cell-mediated immunity, mainly a decrease in the number and function of $\mathrm{CD}^{+}$lymphocytes, which occurs in people infected with the human immunodeficiency virus (HIV), is the major risk factor for Pneumocystis pneumonia. Among people living with HIV, P. jirovecii (D in Figure) persists as the most common cause of serious and often fatal respiratory infection, that continues to be a life-threatening defining illness in patients with acquired immunodeficiency syndrome (AIDS). ${ }^{(38)}$ Yearly, more than 400,000 cases of Pneumocystis pneumonia are estimated to occur world- 
wide, with mortality rates ranging from $10-60 \%$ or higher, depending on the patient population, comorbidities and time for diagnosis. ${ }^{(10,38)}$ Unlike other fungal species affecting humans, evidence suggests that $P$. jirovecii colonises the respiratory tract of asymptomatic individuals and that person-to-person transmission is the most likely mode of infection, which is of clinical significance as colonised individuals may be at risk of development pneumonia or may transmit Pneumocystis to others. ${ }^{(38,39)}$

Low $\mathrm{CD}^{+}$counts is also the most important risk factor to acquire infection by $C$. neoformans, an environmental encapsulated yeast. ${ }^{(40)}$ As $C$. neoformans has a special predilection for the central nervous system (CNS), the main presentation of cryptococcosis is meningoencephalitis, which if left untreated, is deadly or leads to long-term neurological sequelae. . $^{(5,41)}$ Although the introduction of antiretroviral treatments (ART) for people living with HIV has reduced the global incidence of cryptococcosis, the limited access to healthcare and the increasing number of HIV-positive people contribute to the high prevalence of cryptococcal infection in lowand middle-income countries. ${ }^{(42)}$ In patients with a $\mathrm{CD} 4^{+}$ count less than 100 cells $/ \mu \mathrm{L}$ who are not receiving effective ART, screening for asymptomatic cryptococcal antigenaemia combined with pre-emptive antifungal therapy with fluconazole has proven to be an effective strategy to prevent symptomatic meningitis whilst reducing the number of deaths. ${ }^{(43)}$ Nevertheless, worldwide there are circa 225,000 estimated new cases of cryptococcal meningitis per year, causing over 180,000 deaths, which accounts for about $15 \%$ of AIDS-associated deaths. ${ }^{(44)}$ In addition, concomitant treatment with antiretroviral drugs in patients with AIDS-related cryptococcosis can cause exaggerated inflammatory response as a result of immune reconstitution inflammatory syndrome, which contributes to even higher mortality rates. ${ }^{(40,45)}$

Histoplasmosis and talaromycosis, caused by the thermal dimorphic fungi Histoplasma capsulatum (E in Figure) and Talaromyces (formerly Penicillium) marnef$f e i$, respectively, are also major opportunistic infections affecting patients with HIV/AIDS, which present mostly as disseminated disease. ${ }^{(42)}$ Disseminated histoplasmosis, reported worldwide with about 100,000 cases occurring every year, has most endemic cases in the Americas, where the incidence has been estimated to reach $25 \%$ of patients with HIV infection, being an AIDS-defining illness in up to $75 \%$ of patients and with mortality rates of almost $60 \%{ }^{(6,42,46)}$ In Latin America, it is estimated that, among people living with HIV, the number of deaths caused by histoplasmosis may be higher than those caused by tuberculosis. In fact, histoplasmosis, which is considered neglected in this region, is often misdiagnosed as tuberculosis ${ }^{(47)}$ Talaromycosis, restricted to southeast Asia, southern China and northeastern India, remains one of the major causes of HIV-associated mortality in these endemic areas, especially in people with undiagnosed and untreated HIV infection. ${ }^{(42,46,48)}$

Uncontrolled diabetes mellitus, particularly in patients having ketoacidosis, is the major predisposing factor for developing mucormycosis, which is the third most common angioinvasive fungal infection following can- didiasis and aspergillosis. ${ }^{(49,50)}$ Mucormycetes species are vasotropic and cause tissue infarctions. ${ }^{(49)}$ Among them, Rhizopus oryzae (F in Figure) is the most frequently recovered species in patients with mucormycosis $(\sim 70 \%)$, followed by Mucor spp., Rhizomucor spp., Syncephalastrum spp., Cunninghamella bertholletiae, Apophysomyces elegans, Lichtheimia spp., and Saksenaea spp., among other numerous species. ${ }^{(51)}$ After inhalation of spores from environmental sources, immunocompromised hosts can develop upper and lower respiratory disease, which if left untreated, can subsequently spread to the CNS causing rhinocerebral mucormycosis. ${ }^{(52)}$ In people with diabetes, monocytes and macrophages are dysfunctional and fail to phagocyte and kill the Mucormycetes spores, allowing their germination and subsequent proliferation as hyphal elements, which leads to rapid invasion of deeper tissues and dissemination. ${ }^{(53)}$ Despite several simultaneous approaches used for treatment, such as aggressive debridement or amputation, antifungal therapy and medical management or correction of the underlying condition, mucormycosis associated mortality rates continue to be very high, reaching almost $100 \%$ among patients with disseminated disease. ${ }^{(49,52)}$

Chronic obstructive pulmonary disease (COPD) represents an important, nonclassical underlying condition for the increasing number of acute invasive pulmonary aspergillosis, with a very high mortality rate. Associated with the pulmonary disease, structural modifications in the lung architecture that favour lung parenchyma invasion and necrosis due to Aspergillus spp., in addition to prolonged use of immunosuppressive treatment, such as corticosteroid therapy, might increase the susceptibility of COPD patients to acquire an IFD ${ }^{(54)}$ Furthermore, recurrent hospitalisation, invasive procedures, broadspectrum antibiotic use and other factors such as tobacco smoke, alcoholism, diabetes mellitus and/or malnutrition could contribute to promote invasive aspergillosis in COPD patients. ${ }^{(54,55)}$

\section{Bacterial and viral co-infections}

Fungal pulmonary infections can be acquired primarily or secondarily during tuberculosis infection. Co-morbidities such as immunodeficiency, mainly due to HIV, chronic illnesses and malignancy, together with inherent factors of the community like poverty and malnutrition, worsen the co-infection and the prognosis of patients. ${ }^{(56)}$ In 2018, it was estimated that globally 10 million people contracted tuberculosis, causing 1.2 million deaths among HIV-negative people and 251,000 deaths associated with HIV infection. ${ }^{(57)}$ As fungal pulmonary disease does not show specific radiological and clinical manifestations, compared to those of pulmonary tuberculosis, misdiagnosis and late or no-antifungal therapy cause high morbidity and mortality rates in co-infected patients. With an incidence that can reach $50 \%$ of cases, depending on the geographical distribution, different fungal pathogens coexist with pulmonary tuberculosis, amplifying the severity of the disease, especially when dissemination occurs. $A$. $f u$ migatus, Aspergillus niger, $H$. capsulatum, C. neoformans and $C$. albicans are often responsible for causing severe secondary infections in tuberculosis patients..$^{(10,56,58,59)}$ 
Reactivation of latent cytomegalovirus (CMV) can lead to the development of concomitant or sequential fungal infections in immunosuppressed patients. Both solid organ and autologous stem cell transplant recipients, with a history of CMV infection, have higher risk for developing IFD. ${ }^{(60,61)}$ In HSCT patients, Pneumocystis pneumonia and invasive mold infections, including invasive aspergillosis, represent the most commonly encountered IFD during the late post-engraftment period. ${ }^{(32,61)} \mathrm{Un}$ treated CMV viremia, which occurs primarily in patients who were seropositive before transplantation and who develop GvHD, is followed by pneumonia that may be accompanied by superinfection with fungal pathogens, leading to substantial morbidity and mortality. ${ }^{(30)}$

A dysfunctional immune response, which can cause severe alveolar lung damage and even systemic pathology, has been reported to occur rarely in people with coronavirus disease 2019 (COVID-19), a viral infection caused by the severe acute respiratory syndrome coronavirus 2 (SARS-CoV-2), first reported in $\mathrm{Wu}-$ han, China, at the end of 2019. ${ }^{(62,63)}$ While unusual, this harmful interaction of SARS-CoV-2 with the immune system of patients with severe COVID-19 pneumonia seems to increase the susceptibility of these patients to acquire secondary infections, including certain mycoses. ${ }^{(62)}$ Although the incidence of IFD associated with COVID-19 is still unknown, Aspergillus infections have been, so far, the most commonly reported fungal co-infections in COVID-19 patients. ${ }^{(64)}$ Invasive aspergillosis, however, has been associated with admission to critical care because of COVID-19 pneumonia and severe acute respiratory syndrome. ${ }^{(65)}$ In addition, most co-infected patients had previous history of underlying immunocompromised status or pre-existing comorbidities, mainly hypertension, diabetes, obesity and haematological malignancies. ${ }^{(65,66)}$ Much less frequently, candidiasis has been also reported as co-infection in hospitalised patients with COVID-19.(67,68,69) However, the contribution of the underlying disease and COVID-19, as attributable risk factors to acquire fungal infections requires careful analysis and to date remains to be determined. ${ }^{(64)}$

\section{Environmental exposure}

Environmental factors play a unique role in the epidemiology of invasive mold infections. Although infrequent, these opportunistic infections are serious complications of both traumatic and post-traumatic injury. ${ }^{(70)}$ Patients can be directly inoculated during tissue injury; alternatively, fungi can infect the tissue after skin trauma, burns or surgery. In all patients, however, cutaneous manifestations are followed by angioinvasion, which results in vessel thrombosis and tissue necrosis. ${ }^{(53)}$ When the patient has any kind of immunosuppression besides the traumatic injury, tissue infarction and necrosis occur usually shortly after the inoculation event. ${ }^{(70)}$ Globally, mucormycosis is the most common fatal complication that can occur after trauma, because of the quick dissemination of spores and deep extension to bone, tendon, or muscle, after skin disruption. ${ }^{(51)}$ Upon inoculation, $R$. oryzae obtains iron from the host, which is abundant in patients with strong haemorrhage and/or receiving blood transfusions. ${ }^{(52)}$ Mucormycetes have been reported causing outbreaks of traumatic implantation of contaminated soil or water after natural disasters, including tsunamis and tornadoes. Motor vehicle collisions and blasts from war-related injuries are also common forms to get severe trauma among the population. ${ }^{(71)}$ After dissemination occurs, even young, previously healthy patients are susceptible to mucormycosis and even with aggressive approaches such as disfiguring debridement or amputation, case-fatality rate can reach $100 \%$. $^{(71,72)}$

In conclusion, concomitant with the increased prevalence of at-risk and immunocompromised people, rates and spectrum of invasive fungal infections are rising and becoming more frequent worldwide. Certainly, opportunities are increasing for opportunistic fungi. Extended survival of patients related to improvements in supportive and intensive care, prolonged treatment of bacterial infections, high-dose chemotherapeutic regimens to treat cancers, global practice of solid organ and stem cell transplantation procedures, increased use of immunosuppressive and biologic therapies, the advent of AIDS, and even natural disasters, among other factors, significantly contribute to the impact of fungal pathogens on human health. Although advances in medical technology, improved diagnostics, earlier administration of antifungal treatments and more effective preemptive therapy have helped reducing morbidity and mortality for most IFD, fungal opportunistic infections persist severe, often underdiagnosed, life-threatening and have a significant economic burden. Therefore, as fungi are ubiquitous in the environment and they are increasingly infecting vulnerable populations worldwide, especially those living in resource-limited settings, there is an urgent need to increase clinical awareness, in order to develop early intervention strategies. Identifying major risk factors or conditions is of utmost importance to prevent IFD and meet the need of decreasing the still staggering high mortality-associated rates. Moreover, even though IFD are among the deadliest communicable diseases worldwide, the vast majority of these mycoses are not reportable to public health authorities in most countries, which represents another challenge and makes IFD less likely to be documented. Whilst waiting for mandatory case reporting, comprehensive eco-epidemiological studies together with voluntary reports from clinicians and laboratories are needed, aimed to improve disease surveillance and to achieve a better understanding of the public health implications of IFD around the world. Obtaining a true picture of the global burden of invasive mycoses, including the patients' associated conditions and risk factors, is key to making decisions and providing inputs into health-care services, programmes, responses and policies, which will ultimately be addressed to improving people's health and wellbeing.

\section{ACKNOWLEDGEMENTS}

To Dr Elizabeth Castañeda and Dr Simon Dingsdag, for their valuable suggestions during the writing of the manuscript. 


\section{AUTHORS' CONTRIBUTION}

CF conceived the study, prepared tables and figures, wrote and revised the manuscript. The author declares no conflict of interest.

\section{REFERENCES}

1. Taylor DL, Hollingsworth TN, McFarland JW, Lennon NJ, Nusbaum C, Ruess RW. A first comprehensive census of fungi in soil reveals both hyperdiversity and fine-scale niche partitioning. Ecol Monogr. 2014;84:3-20.

2. Govorushko S, Rezaee R, Dumanov J, Tsatsakis A. Poisoning associated with the use of mushrooms: A review of the global pattern and main characteristics. Food Chem Toxicol. 2019;128:267-79.

3. Brown GD, Denning DW, Levitz SM. Tackling human fungal infections. Science. 2012;336(6082):647.

4. Casadevall A, Burnham CA, Cassell GH, Dermody TS, Enquist L. 2019. One health: fungal pathogens of humans, animals, and plants. Colloquium report. American Academy of Microbiology Colloquium. 2017 Oct 18; Washington, DC, USA.

5. Kohler JR, Hube B, Puccia R, Casadevall A, Perfect JR. Fungi that infect humans. Microbiol Spectr. 2017;5(3):FUNK-0014-2016.

6. Bongomin F, Gago S, Oladele RO, Denning DW. Global and multi-national prevalence of fungal diseases-estimate precision. J Fungi (Basel).2017;3(4):57.

7. Donnelly JP, Chen SC, Kauffman CA, Steinbach WJ, Baddley JW, Verweij PE, et al. Revision and update of the consensus definitions of invasive fungal disease from the European Organization for Research and Treatment of Cancer and the Mycoses Study Group Education and Research Consortium. Clin Infect Dis. 2019;71(6):1367-76.

8. Badiee P, Hashemizadeh Z. Opportunistic invasive fungal infections: diagnosis \& clinical management. Indian J Med Res. 2014;139(2):195-204.

9. Schmiedel Y, Zimmerli S. Common invasive fungal diseases: an overview of invasive candidiasis, aspergillosis, cryptococcosis, and Pneumocystis pneumonia. Swiss Med Wkly. 2016;146:w14281.

10. Brown GD, Denning DW, Gow NA, Levitz SM, Netea MG, White TC. Hidden killers: human fungal infections. Sci Transl Med.2012;4(165):165rv113.

11. Perlroth J, Choi B, Spellberg B. Nosocomial fungal infections: epidemiology, diagnosis, and treatment. Med Mycol. 2007;45(4):321-46.

12. McCarty TP, Pappas PG. Invasive candidiasis. Infect Dis Clin North Am. 2016;30(1):103-24.

13. Seelig MS. The role of antibiotics in the pathogenesis of Candida infections. Am J Med. 1966;40(6):887-917.

14. Cavalheiro M, Teixeira MC. Candida biofilms: threats, challenges, and promising strategies. Front Med (Lausanne). 2018;5:28.

15. Ramage G, Rajendran R, Sherry L, Williams C. Fungal biofilm resistance. Int J Microbiol. 2012;2012:528521.

16. Kauffman CA, Malani AN. Fungal infections associated with contaminated steroid injections. Microbiol Spectr. 2016;4(2):EI100005-2015.

17. Pettit AC, Kropski JA, Castilho JL, Schmitz JE, Rauch CA, Mobley $\mathrm{BC}$, et al. The index case for the fungal meningitis outbreak in the United States. N Engl J Med. 2012;367(22):2119-25.

18. Orsini M, Otaiza F, Vega P, Hederra LM, Pidal P, Salas V, et al. Outbreak of fungemias by Sarocladium kiliense in eight public hospitals per intrinsic contamination of ondansetron intravenous. Rev Chilena Infectol. 2018;35(4):363-70.
19. Etienne KA, Roe CC, Smith RM, Vallabhaneni S, Duarte C, Escadon $\mathrm{P}$, et al. Whole-genome sequencing to determine origin of multinational outbreak of Sarocladium kiliense bloodstream infections. Emerg Infect Dis. 2016;22(3):476-81.

20. Abers MS, Ghebremichael MS, Timmons AK, Warren HS, Poznansky MC, Vyas JM. A critical reappraisal of prolonged neutropenia as a risk factor for invasive pulmonary aspergillosis. Open Forum Infect Dis. 2016;3(1):ofw036.

21. Gazendam RP, van Hamme JL, Tool AT, Hoogenboezem M, van den Berg JM, Prins JM, et al. Human neutrophils use different mechanisms to kill Aspergillus fumigatus conidia and hyphae: evidence from phagocyte defects. J Immunol. 2016;196(3):1272-83.

22. Shopova IA, Belyaev I, Dasari P, Jahreis S, Stroe MC, Cseresnyes $\mathrm{Z}$, et al. Human neutrophils produce antifungal extracellular vesicles against Aspergillus fumigatus. mBio. 2020;11(2):e00596-20.

23. Muhlemann K, Wenger C, Zenhausern R, Tauber MG. Risk factors for invasive aspergillosis in neutropenic patients with hematologic malignancies. Leukemia. 2005;19(4):545-50.

24. Baden LR, Swaminathan S, Angarone M, Blouin G, Camins BC, Casper C, et al. Prevention and treatment of cancer-related infections, version 2.2016, NCCN Clinical Practice Guidelines in Oncology. J Natl Compr Canc Netw. 2016;14(7):882-913.

25 Neofytos D, Fishman JA, Horn D, Anaissie E, Chang CH, Olyaei A, et al. Epidemiology and outcome of invasive fungal infections in solid organ transplant recipients. Transpl Infect Dis. 2010;12(3):220-9.

26. Pappas PG, Alexander BD, Andes DR, Hadley S, Kauffman CA, Freifeld A, et al. Invasive fungal infections among organ transplant recipients: results of the Transplant-Associated Infection Surveillance Network (TRANSNET). Clin Infect Dis. 2010;50(8):1101-11.

27. Baddley JW. Clinical risk factors for invasive aspergillosis. Med Mycol. 2011;49 Suppl 1:S7-S12.

28. Anesi JA, Baddley JW. Approach to the solid organ transplant patient with suspected fungal infection. Infect Dis Clin North Am. 2016;30(1):277-96.

29. Kontoyiannis DP, Marr KA, Park BJ, Alexander BD, Anaissie EJ, Walsh TJ, et al. Prospective surveillance for invasive fungal infections in hematopoietic stem cell transplant recipients, 2001-2006: overview of the Transplant-Associated Infection Surveillance Network (TRANSNET) Database. Clin Infect Dis. 2010;50(8):1091-1100.

30. Wingard JR, Hsu J, Hiemenz JW. Hematopoietic stem cell transplantation: an overview of infection risks and epidemiology. Infect Dis Clin North Am. 2010;24(2):257-72.

31. Neofytos D, Horn D, Anaissie E, Steinbach W, Olyaei A, Fishman $\mathrm{J}$, et al. Epidemiology and outcome of invasive fungal infection in adult hematopoietic stem cell transplant recipients: analysis of Multicenter Prospective Antifungal Therapy (PATH) Alliance registry. Clin Infect Dis. 2009;48(3):265-73.

32. Neofytos D. Antimicrobial prophylaxis and preemptive approaches for the prevention of infections in the stem cell transplant recipient, with analogies to the hematologic malignancy patient. Infect Dis Clin North Am. 2019;33(2):361-80.

33. Pagano L, Caira M, Candoni A, Offidani M, Fianchi L, Martino $\mathrm{B}$, et al. The epidemiology of fungal infections in patients with hematologic malignancies: the SEIFEM-2004 study. Haematologica. 2006;91(8):1068-75.

34. Davis MR, Thompson GR 3rd, Patterson TF. Fungal infections potentiated by biologics. Infect Dis Clin North Am. 2020;34(2):389-411. 
35. Baddley JW, Cantini F, Goletti D, Gomez-Reino JJ, Mylonakis E, San-Juan R, et al. ESCMID Study Group for Infections in Compromised Hosts (ESGICH) Consensus Document on the safety of targeted and biological therapies: an infectious diseases perspective (Soluble immune effector molecules [I]: anti-tumor necrosis factoralpha agents). Clin Microbiol Infect. 2018;24 Suppl 2:S10-S20.

36. Wallis RS, Broder MS, Wong JY, Hanson ME, Beenhouwer DO. Granulomatous infectious diseases associated with tumor necrosis factor antagonists. Clin Infect Dis. 2004;8(9):1261-5.

37. Baddley JW, Winthrop KL, Chen L, Liu L, Grijalva CG, Delzell E, et al. Non-viral opportunistic infections in new users of tumour necrosis factor inhibitor therapy: results of the SAfety Assessment of Biologic ThERapy (SABER) study. Ann Rheum Dis. 2014;73(11):1942-8

38. Krajicek BJ, Thomas CF Jr, Limper AH. Pneumocystis pneumonia: current concepts in pathogenesis, diagnosis, and treatment. Clin Chest Med. 2009;30(2):265-78, vi.

39. Morris A, Wei K, Afshar K, Huang L. Epidemiology and clinical significance of Pneumocystis colonization. J Infect Dis. 2008;197(1):10-7.

40. Price MS, Perfect JR. Host defenses against cryptococcosis. Immunol Invest. 2011;40(7-8):786-808

41. Jarvis JN, Bicanic T, Loyse A, Namarika D, Jackson A, Nussbaum JC, et al. Determinants of mortality in a combined cohort of 501 patients with HIV-associated cryptococcal meningitis: implications for improving outcomes. Clin Infect Dis. 2014;58(5):736-45.

42. Limper AH, Adenis A, Le T, Harrison TS. Fungal infections in HIV/AIDS. Lancet Infect Dis. 2017;17(11):e334-e43.

43. Meya DB, Manabe YC, Castelnuovo B, Cook BA, Elbireer AM, Kambugu A, et al. Cost-effectiveness of serum cryptococcal antigen screening to prevent deaths among HIV-infected persons with a CD4+ cell count $<$ or $=100$ cells $/$ microL who start HIV therapy in resource-limited settings. Clin Infect Dis. 2010;51(4):448-55.

44. Rajasingham R, Smith RM, Park BJ, Jarvis JN, Govender NP, Chiller TM, et al. Global burden of disease of HIV-associated cryptococcal meningitis: an updated analysis. Lancet Infect Dis. 2017;17(8):873-81.

45. Haddow LJ, Colebunders R, Meintjes G, Lawn SD, Elliott JH, Manabe YC, et al. Cryptococcal immune reconstitution inflammatory syndrome in HIV-1-infected individuals: proposed clinical case definitions. Lancet Infect Dis. 2010;10(11):791-802.

46. Chastain DB, Henao-Martinez AF, Franco-Paredes C. Opportunistic invasive mycoses in AIDS: cryptococcosis, histoplasmosis, coccidiodomycosis, and talaromycosis. Curr Infect Dis Rep. 2017;19(10):36.

47. Adenis AA, Valdes A, Cropet C, McCotter OZ, Derado G, Couppie $\mathrm{P}$, et al. Burden of HIV-associated histoplasmosis compared with tuberculosis in Latin America: a modelling study. Lancet Infect Dis.2018;18(10):1150-9.

48. Vanittanakom N, Cooper CR Jr, Fisher MC, Sirisanthana T. Penicillium marneffei infection and recent advances in the epidemiology and molecular biology aspects. Clin Microbiol Rev. 2006;19(1):95-110.

49. Petrikkos G, Skiada A, Lortholary O, Roilides E, Walsh TJ, Kontoyiannis DP. Epidemiology and clinical manifestations of mucormycosis. Clin Infect Dis. 2012;54 Suppl 1:S23-34.

50. Torres-Narbona M, Guinea J, Munoz P, Bouza E. Zygomycetes and zygomycosis in the new era of antifungal therapies. Rev Esp Quimioter. 2007;20(4):375-86.

51. Roden MM, Zaoutis TE, Buchanan WL, Knudsen TA, Sarkisova TA, Schaufele RL, et al. Epidemiology and outcome of zygomycosis: a review of 929 reported cases. Clin Infect Dis. 2005;1(5):634-53
52. Ibrahim AS, Spellberg B, Walsh TJ, Kontoyiannis DP. Pathogenesis of mucormycosis. Clin Infect Dis. 2012;54 Suppl 1:S16-22.

53. Mantadakis E, Samonis G. Clinical presentation of zygomycosis. Clin Microbiol Infect. 2009;15 Suppl 5:15-20.

54. Bulpa P, Dive A, Sibille Y. Invasive pulmonary aspergillosis in patients with chronic obstructive pulmonary disease. Eur Respir J. 2007;30(4):782-800.

55. Kousha M, Tadi R, Soubani AO. Pulmonary aspergillosis: a clinical review. Eur Respir Rev.2011;20(121):156-74.

56. Amiri MRJ, Siami R, Khaledi A. Tuberculosis status and coinfection of pulmonary fungal infections in patients referred to reference laboratory of Health Centers Ghaemshahr City during 20072017. Ethiop J Health Sci. 2018;28(6):683-90.

57. World Health Organization (WHO). Global tuberculosis report 2019. Geneva: WHO; 2019.

58. Bansod S, Rai M. Emerging of mycotic infection in patients infected with Mycobacterium tuberculosis. World J Med Sci. 2008;3(2):74-80.

59. Osman NM, Gomaa AA, Sayed NM, Abd el aziz AA. Microarray detection of fungal infection in pulmonary tuberculosis. Egypt $\mathrm{J}$ Chest Dis Tuberc. 2013;62(1):151-7.

60. Yong MK, Slavin MA, Kontoyiannis DP. Invasive fungal disease and cytomegalovirus infection: is there an association? Curr Opin Infect Dis. 2018;31(6):481-9.

61. Marchesi F, Pimpinelli F, Di Domenico EG, Renzi D, Gallo MT, Regazzo G, et al. Association between CMV and invasive fungal infections after autologous stem cell transplant in lymphoproliferative malignancies: opportunistic partnership or cause-effect relationship? Int J Mol Sci. 2019;20(6):1373.

62. Tay MZ, Poh CM, Renia L, MacAry PA, Ng LFP. The trinity of COVID-19: immunity, inflammation and intervention. Nat Rev Immunol. 2020;20(6):363-74.

63. Zhu N, Zhang D, Wang W, Li X, Yang B, Song J, et al. A novel coronavirus from patients with pneumonia in China, 2019. N Eng1 J Med. 2020;382(8):727-33.

64. Gangneux JP, Bougnoux ME, Dannaoui E, Cornet M, Zahar JR. Invasive fungal diseases during COVID-19: We should be prepared. J Mycol Med. 2020;30(2):100971.

65. Mohamed A, Rogers TR, Talento AF. COVID-19 associated invasive pulmonary aspergillosis: diagnostic and therapeutic challenges. J Fungi (Basel). 2020;6(3):115.

66. Falces-Romero I, Ruiz-Bastian M, Diaz-Pollan B, Maseda E, Garcia-Rodriguez J, Group SA-C-W. Isolation of Aspergillus spp. in respiratory samples of patients with COVID-19 in a Spanish tertiary care hospital. Mycoses. 2020. doi: 10.1111/myc,13155. Online ahead of print.

67. Lai CC, Wang CY, Hsueh PR. Co-infections among patients with COVID-19: The need for combination therapy with non-anti-SARS-CoV-2 agents? J Microbiol Immunol Infect. 2020;53(4):505-12.

68. Hughes S, Troise O, Donaldson H, Mughal N, Moore LSP. Bacterial and fungal coinfection among hospitalized patients with COVID-19: a retrospective cohort study in a UK secondary-care setting. Clin Microbiol Infect. 2020;26(10):1395-9.

69. Song G, Liang G, Liu W. Fungal co-infections associated with global COVID-19 pandemic: a clinical and diagnostic perspective from China. Mycopathologia. 2020;185(4):599-606.

70. Kronen R, Liang SY, Bochicchio G, Bochicchio K, Powderly WG, Spec A. Invasive fungal infections secondary to traumatic injury. Int J Infect Dis. 2017;62:102-11. 
71. Zahoor B, Kent S, Wall D. Cutaneous mucormycosis secondary to penetrative trauma. Injury. 2016;47(7):1383-7.

72. Skiada A, Rigopoulos D, Larios G, Petrikkos G, Katsambas A. Global epidemiology of cutaneous zygomycosis. Clin Dermatol. 2012;30(6):628-32.
73. Public Health Image Library (PHIL), Centers for Disease Control and Prevention (CDC) [Internet]. [cited 2020 Aug 15]. Available from: https://phil.cdc.gov/default.aspx

74. Global Action Fund for Fungal Infections (GAFFI) [Internet]. [cited 2020 Sep 21]. Available from: https://www.gaffi.org/why/ fungal-disease-frequency/ 